\title{
Multiple Views Gait Recognition using View Transformation Model Based on Optimized Gait Energy Image
}

\author{
Worapan Kusakunniran ${ }^{1,4}$, Qiang $\mathrm{Wu}^{2}$, Hongdong $\mathrm{Li}^{3,4}$, and Jian Zhang ${ }^{1,4}$ \\ ${ }^{1}$ University of New South Wales, ${ }^{2}$ University of Technology Sydney, \\ ${ }^{3}$ Australian National University, and ${ }^{4}$ National ICT Australia-NICTA \\ \{worapan.kusakunniran@nicta.com.au, wug@it.uts.edu.au, \\ hongdong.li@nicta.com.au, and jian.zhang@nicta.com.au\}
}

\begin{abstract}
Gait is one of well recognized biometrics that has been widely used for human identification. However, the current gait recognition might have difficulties due to viewing angle being changed. This is because the viewing angle under which the gait signature database was generated may not be the same as the viewing angle when the probe data are obtained. This paper proposes a new multi-view gait recognition approach which tackles the problems mentioned above. Being different from other approaches of same category, this new method creates a so called View Transformation Model (VTM) based on spatial-domain Gait Energy Image (GEI) by adopting Singular Value Decomposition (SVD) technique. To further improve the performance of the proposed VTM, Linear Discriminant Analysis (LDA) is used to optimize the obtained GEI feature vectors. When implementing SVD there are a few practical problems such as large matrix size and over-fitting. In this paper, reduced SVD is introduced to alleviate the effects caused by these problems. Using the generated VTM, the viewing angles of gallery gait data and probe gait data can be transformed into the same direction. Thus, gait signatures can be measured without difficulties. The extensive experiments show that the proposed algorithm can significantly improve the multiple view gait recognition performance when being compared to the similar methods in literature.
\end{abstract}

\section{Introduction}

Gait is one of the well recognized biometric features to re-identify a human at a distance from a camera. A large number of successful gait recognition techniques have been continuously contributed. However, appearance changes of individuals due to viewing angle changes cause many difficulties for 2D appearance-based gait recognition. This situation cannot be easily avoided in a practical surveillance application. There are three major approach categories to sort out the problem, namely; 1) seeking a gait feature invariant to view change; 2) reconstructing gait under any viewing angle using $3 \mathrm{D}$ structure information which is obtained by calibration; 3) projecting gait feature from one viewing angle to the other by using view transformation.

In the approaches of the first category, Jean et al. [3] introduced a method to compute and evaluate viewnormalized trajectories of pedestrian body parts obtained from monocular video sequences. It used $2 \mathrm{D}$ trajectories of feet and head from tracked silhouettes to segment the walking trajectory into piecewise linear segments. On each linear segments, a normalization process was carried out for head and feet trajectories. Thus, head and feet trajectories always appear like being seen from a fronto-parallel viewpoint. A gait feature was obtained based on this kind of pre-processing. Such feature is invariant to viewing angle changes. However, this technique was fine only for a limited range of viewing angles. This is because that the duration of self-occlusion intervals is not longer than the duration of time intervals where both feet are visible. Kale et al. [4] developed a method to synthesize images of arbitrary-view from an image under a particular view through perspective projection in a sagittal plane. This approach does not work well because of a self occlusion when an angle formed by image plane and the sagittal plane is large.

Alternatively, view changes problem can be solved by integrating 3D structure information from multiple cameras. Bodor et al. [1] used image-based rendering techniques to reconstruct gait for any required viewing angle. It combined several gaits which were acquired by various cameras under the different views. This combimation produced a synthetic gait whose viewing angle was the same as the viewing angle of training gait dataset. Shakhnarovich et al. [10] and Lee [6] synthesized an image for a virtual viewing angle using a visual hull. Although the synthetic images constructed by this catagory of approaches would be more precice, they require complicated setup of a cooperating multi-camera system.

Being different from the first two categories, the third approach category is to establish View Transformation Model (VTM) through a factorization process [7] [12]. The method proposed in [7] was to first obtain frequency fea- 
tures on each position across an image sequence by Fourier operation. The significant Fourier components were then selected and used to build up a VTM. Instead of using Fourier feature, [12] created VTM using static image on spatial domain directly. Both methods [7] and [12] could transform any gait features from one viewing angle to the other using VTM without catching the special invariant gait features as described in the first category. Compare to the second approach category, rather than constructing expensive cooperating multi-camera system, the third category adopt factorization e.g. Singular Value Decomposition (SVD) to realize view transformation.

However, it can be seen that gait image synchronization in [12] was sensitive. Small error from synchronization could cause large variation in construction of VTM. For the paper [7], the method was still immature since gait recognition rate under multiple view was low based on the proposed method. This paper proposes a new multiple view gait recognition approach which will tackles the constrains presented in [7] and [12]. It will improve the recognition performance in terms of accuracy and computational time.

The proposed method is first to construct GEI from complete walking cycle(s) using a reliable normalization process [5]. Then, Linear Discriminant Analysis (LDA) is carried out to optimize GEI features. Next, by using SVD, VTM is formulated based on GEI features extracted from multiple viewing angles and multiple subjects. Reduced version of SVD is introduced to avoid over-sizing and overfitting of the model. In the recognition stage, using the constructed VTM, viewing angles of gallery gait data and probe gait data can be transformed into the same direction. Then, gait similarity is measured without difficulties.

Another benefit from the proposed method is that it can independently train the gait signature database for common gait recognition and construct View Transformation Model for viewing angle projection. That is, it can use the separate datasets for these two purposes above. To construct a more accurate VTM, it is practically workable to invite enough experience actors to perform the require walking actions under a fully controlled environment. In that way, it can obtain precise gait data from many subjects and on every required viewing angle. Using the proposed method, it can construct a good VTM which can cover a very dense viewing angle range such as $\left(0^{\circ}, 10^{\circ}, 20^{\circ}, \ldots, 180^{\circ}\right)$. For gait recognition purpose, gait signature dataset can be established using normal training procedure for all interesting subjects under various viewing angles. In testing stage, depending on the viewing angle of probe data detected, the created VTM can transform its viewing angle to any other view to match the data in gallery dataset.

The rest of this paper is organized as follows. The methods used to synthesize gait feature are described in section 2. The construction of a view transformation model (VTM) and gait similarity measurement are explained in section 3 and section 4 respectively. Experimental results are analyzed in section 5 and conclusion is drawn in section 6.

\section{Gait Feature Extraction}

Human walking pattern can be considered as periodic action. One gait period contains a sequence of walking poses. To avoid the synchronization difficulties and to prevent noises from individual image, Gait Energy Image (GEI) is created based on the gait sequence in a few walking periods. GEI contains the continuous changes of pose when human walking. GEI holds several key features of human gait including motion frequency, temporal and spatial changes of human body, and global body shape statistic. It well reflects the gait rhythm. In order to create an accurate GEI, it is necessary to include the gait sequence of one period or several periods. Thus, it is required to estimate the walking period.

\subsection{Gait Period Estimation}

This paper adopts the similar method as that one in [13] to determine the period of each gait sequence. The basic procedure is illustrated in Figure 1. It is a complicated procedure for the case of frontal view video sequence.
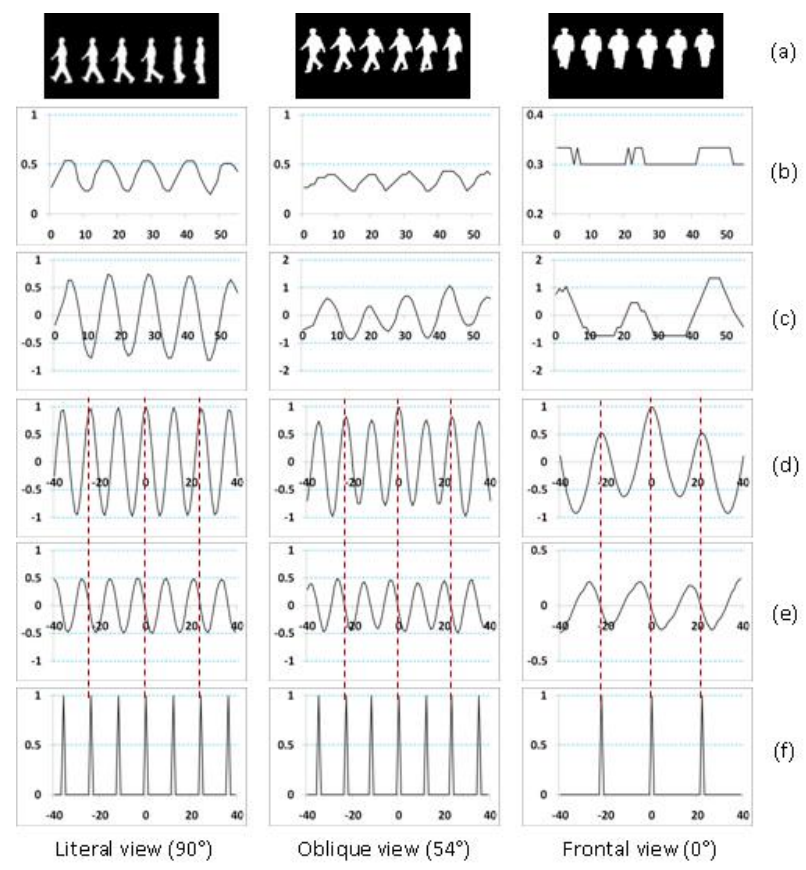

Figure 1. Gait period estimation. (a) Sample silhouette sequences of walking people. (b) Aspect ratio of silhouette bounding box. (c) Normalization of aspect ration followed by mean filtering. (d) Autocorrelation of aspect ration under various offset. (e) First order derivative based on (d). (f) Peak positions indicating the periods.

Figure 1(a) shows three samples of silhouette sequences obtained under the side view, oblique view and front view respectively. Along the time of image sequences, the aspect ratio of each silhouette bounding box is recorded (Figure 1(b)). In order to more precisely estimate the gait period, 
the curves in Figure 1(b) are further processed by deleting the mean value and normalized by its standard deviation. Then, the results are processed by mean filtering (Figure 1(c)). Autocorrelation based on the the results in Figure 1(c) are calculated. The correlation between the curve in Figure 1(c) and the curve by shifting itself in some degree is calculated. The offset range $t$ in this paper is $-40 \leq t \leq 40$ as shown in Figure 1(d). From Figure (b) and (c), it can be seen that the change of bounding box aspect ration is a kind of periodic signal. Thus, when calculating autocorrelation in Figure 1(d), there will be a peak if the offset equals to a gait period or its integer multiple. To more clearly identify the period transition position, first order derivative (Figure 1(e)) is calculated based on the results of Figure 1(d). The period transition position is defined at the zero crossing point along the positive-to-negative direction. According to [13], because of the bilateral symmetry of human gait under any viewing angle except frontal view, the autocorrelation signals sometime contain minor peaks located half way between each pair of consecutive major peaks. So the final period transition positions are shown and aligned using dash lines on Figure 1(f).

\subsection{Different Gait Features}

In this paper, two different methods of generating gait signatures are described. The first one is based on [7]. The second one is our proposed method that captures the gait information in spatial domain through GEI. Supposing that a gait sequence contains $N$ sub-sequence. Each subsequence corresponds to a gait period with $T$ frames. Then, $S_{n, t}(x, y)$ is a particular pixel located at position $(x, y)$ of $t^{t h}(t=1,2, \ldots, T)$ image from $n^{t h}(n=1,2, \ldots, N)$ gait cycle of a walking sequence.

Feature 1: Frequency-domain representation of Gait feature (FG) [7]

All silhouettes are normalized to same heights. The upper half of silhouettes are aligned along horizontal centroid [2]. Then, each bounding box of silhouette is $W \times H$. 1D Fourier Transform is carried out at each position of $(x, y)$ on $S_{n, t}(x, y)$ across all frames (along the time sequence) [7] [8]. Then FG is extracted by computing 1D Fourier transform along a time axis based on gait periodicity [7] [8]. Therefore, the average Discrete Fourier Transform (DFT) of gait feature of $\mathrm{N}$ gait cycles is:

$$
\operatorname{DFT}(x, y, k)=\sum_{n=1}^{N} \sum_{t=1}^{T}\left(S_{n, t}(x, y) e^{(-2 \pi j k t) / T}\right) / N
$$

Then, FG is defined as an amplitude spectrum for DFT:

$$
F G(x, y, k)=|D F T(x, y, k)|
$$

The DC component $(k=0)$ of the DFT is ignored because it does not represent gait periodicity. In addition, the high frequency components contain less intensity than the lower frequency elements. Thus, they are also removed. Figure 2 shows examples of FG feature $(k=1,2, \ldots, 5)$ for various viewing angles of three subjects.
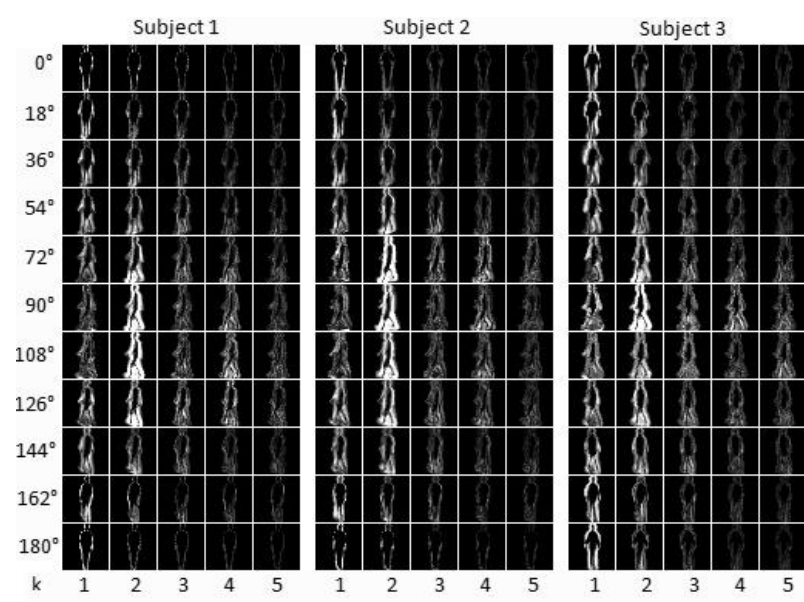

Figure 2. Frequency-domain gait features for various viewing angles.

In this study, only first three frequency components $(k=$ $1,2,3)$ are selected. The actual representation of FG feature is $1 \mathrm{D}$ vector, $f_{k, F G}^{m}$, by concatenating three frequency components of each position along all consecutive rows, where $m$ represents the $m^{t h}$ subject and $k$ represents the $k^{t h}$ viewing angle. Finally, the dimension of $f_{k, F G}^{m}$ is $3 \times W \times H$.

\section{Feature 2: Optimized Gait Energy Image (GEI) Feature}

Rather than normalization along horizontal direction as in feature 1, all silhouettes $S_{n, t}$ are rescaled along both horizontal direction and vertical direction to a fixed size i.e. the same width $(W)$ and the same height $(H)$. Based on the study in [5], this straightforward method can efficiently capture a gait information. GEI feature is obtained as:

$$
G E I(x, y)=\sum_{n=1}^{N} \sum_{t=1}^{T} S_{n, t}(x, y) /(N+T)
$$

Figure 3 shows the examples of GEI under various viewing angles of four different subjects.

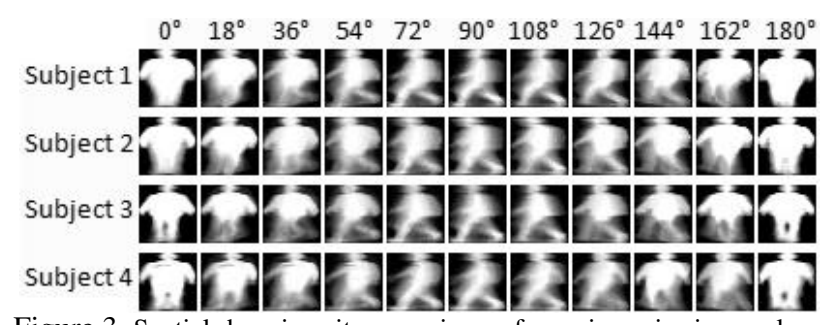

Figure 3. Spatial-domain gait energy image for various viewing angles.

Firstly similar to FG, the actual representation of GEI feature is $1 \mathrm{D}$ vector, $f_{k, G E I}^{m}$, by concatenating the value of each position (Equation (3)) along all consecutive rows, where $m$ represents the $m^{t h}$ subject and $k$ represents the $k^{t h}$ viewing angle. Thus, the dimension of $f_{k, G E I}^{m}$ is $W \times H$. 
Secondly, a Linear Discriminant Analysis (LDA) to spatial-domain GEI (Figure 3) is applied to acquire an optimized version of the feature. There are two main advantages of using LDA. The first benefit is to significantly reduce dimension of gait feature, which is very useful aspect in real time application. The second benefit is to maximize a margin between gait features from different subjects and as well to minimize a distance of gait features that belong to the same subject.

Theoretically, LDA is a classical statistical approach used for supervised dimensionality reduction, classification, and feature extraction [9]. It computes an optimal projection by searching the directions for maximum discrimination of classes. The projection is done by minimizing the trace of within-class scatter matrix $\left(S_{w}\right)$ and maximizing the trace of between-class scatter matrix $\left(S_{b}\right)$ simultaneously. However, since scatter matrices are proportional to the covariance matrix $\left(S_{t}\right), S_{t}=S_{b}+S_{w}$, LDA process is equivalent to minimizing the trace of $S_{t}$ and maximizing the trace of $S_{b}$ simultaneously. Therefore, LDA considers maximizing of the following objective [14]:

$$
J(w)=\frac{w^{T} S_{b} w}{w^{T} S_{t} w}
$$

According to Equation (4), the optimal transformation $(w)$ in LDA can be constructed from a process of Eigendecomposition on between-class scatter matrix $\left(S_{b}\right)$ and covariance matrix $\left(S_{t}\right)$. This means $w$ is an eigenvector of $\left(S_{t}\right)^{-1} S_{b}$, where separation factor is equal to the corresponding eigenvalue. Thus, to reduce feature dimension to $k$, the eigenvectors corresponding to the $k$ largest eigenvalues are selected.

As mentioned above, by applying LDA on GEI, our approach can achieve the reduction of impact from gait feature's geometric properties and gait feature's dimension. Therefore, the optimized GEI is expected to be better factorized in process of VTM construction, being compared to original spatial-domain GEI.

\section{Construction of View Transformation Model}

This section describes the construction of view transformation model (VTM) using a Truncated Singular Value Decomposition (TSVD) technique. The VTM is developed based on our proposed gait feature, that is, the optimized GEI. It can be seen in Section 5 that the performance of VTM using the proposed feature is better than the VTM described in [7] [12].

\subsection{Singular Value Decomposition (SVD)}

A gait matrix is created as the left hand side matrix in Equation (5). Each row contains gait information from same viewing angle but from the different subjects. Each column contains gait information from same subject but under the different viewing angles. There are total $\mathrm{K}$ viewing angles and M subjects for constructing VTM. $g_{k}^{m}$ denotes $f_{k, F G}^{m}$ or $f_{k, G E I}^{m}$ depending on the features adopted. Assuming that dimension of $g_{k}^{m}$ is $N_{g}$. The factorization process by SVD is as below:

$$
\left[\begin{array}{ccc}
g_{1}^{1} & \ldots & g_{1}^{M} \\
\cdot & \cdot & \cdot \\
\cdot & \cdot & \cdot \\
g_{K}^{1} & \cdot & \cdot \\
g_{K}^{M}
\end{array}\right]=U S V^{T}=\left[\begin{array}{c}
P_{1} \\
\cdot \\
\cdot \\
\cdot \\
P_{K}
\end{array}\right]\left[\begin{array}{lll}
v^{1} & \ldots & v^{M}
\end{array}\right]
$$

where $U$ is the $K N_{g} \times M$ orthogonal matrix. $V$ is the $M \times$ $M$ orthogonal matrix. $S$ is the $M \times M$ diagonal matrix contains the singular values. $P=\left[P_{1}, \ldots, P_{K}\right]^{T}=U S$ where $P_{k}$ is the $N_{g} \times M$ sub-matrix of $U S$. $v^{m}$ is the $M$ dimensional column vector.

The vector $v^{m}$ is an intrinsic gait feature of $m^{t h}$ subject for any viewing angle. $P_{k}$ is a projection matrix which can project intrinsic vector $v$ to the gait feature vector under a specific viewing angle $k . P_{k}$ is independent of the subject. From Equation (5), it can be seen that:

$$
g_{k}^{m}=P_{k} v^{m}
$$

Thus, gait feature transformation from viewing angle $j$ to $i$ is obtained by:

$$
g_{i}^{m}=P_{i} P_{j}^{+} g_{j}^{m}
$$

where $P_{j}^{+}$is the pseudo inverse matrix of $P_{j}$.

\subsection{Optimized View Transformation Model}

In order to simplify SVD computational complexity and make it less sensitive to trivial elements, reduced version of SVD is adopted. There are three main advantages of using reduced version of SVD. Firstly, it reduces the dimension of the view transformation model. Therefore, it makes the algorithm perform faster. Secondly, it is more economic for storage. Thirdly, it improves the accuracy of view transformation process because it avoids over fitting problem in some how by removing the less important elements from the transformation model. These three benefits become significant when there are sufficient training data for constructing VTM.

There are several reduced SVD solutions. TSVD [11] is an efficient one and selected in this study. In Equation 5 , it can be seen that $S$ is a diagonal matrix containing the singular values as follows:

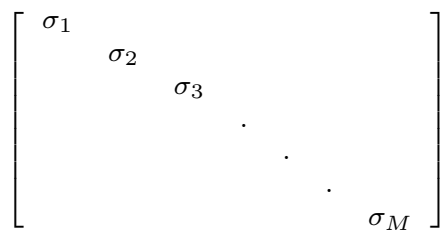


where $\sigma_{1} \geq \sigma_{2} \geq \ldots \geq \sigma_{M} \geq 0$ are the singular values of the matrix $S$. Its rank is $M$. Then, the reduced rank approximation for TSVD is by setting all but the first $r(r<M)$ largest singular values to zero. Thus, the dimension of $P_{k}$ in Equation (5) is reduced from $N_{g} \times M$ to $N_{g} \times r$. The other significant aspect is that such optimization avoids over fitting problem by removing the less important elements from the transformation model.

In the experiments, it can be seen that using Equation (7) is not precise enough for single viewing angle transformation from one to other. This is because that the orthogonality is degenerated when processing silhouette image. To overcome this weakness, gait feature estimated from more than one viewing angles is considered as:

$$
g_{i}^{m}=P_{i}\left[\begin{array}{c}
P_{j(1)} \\
\cdot \\
\cdot \\
\cdot \\
P_{j(k)}
\end{array}\right]^{+}\left[\begin{array}{c}
g_{j(1)}^{m} \\
\cdot \\
\cdot \\
\cdot \\
g_{j(k)}^{m}
\end{array}\right]
$$

In a practical system, gallery gait signature database contains subject's gait features from many predefined viewing angles. However, these viewing angles are not necessary to be the same as the probe data where the viewing angles are obtained. Before measuring the similarity between gallery data and probe data, VTM transforms relevant gallery data from their original views to the views same as the probe data detected. Such kind of assumption is valid in most of cases.

\section{Gait Similarity Measurement}

This paper focuses on comparing the performance of our new approach for VTM construction with the existing approach. Regarding similarity measurements, the simple but widely adopted Euclidean distance is used in our study. Once two gait features under the same viewing angle, $g^{i}$ and $g^{j}$, are obtained. The similarity of the two features $\left(g^{i}, g^{j}\right)$ is linearly measured as follows:

$$
d\left(g^{i}, g^{j}\right)=\sum_{n=1}^{N}\left|g^{i}(n)-g^{j}(n)\right|
$$

where $d\left(g^{i}, g^{j}\right)$ is a distance between gait signatures $g^{i}$ and $g^{j} . N$ is dimension of gait feature. The smaller value of $d$, the more possibility that gait signatures $g^{i}$ and $g^{j}$ belong to the same subject.

\section{Experiments}

\subsection{Datasets}

The experiments use the publically available CASIA gait database B. It is a large multi-view gait dataset that contains 124 subjects. The gait data was captured from 11 views, $0^{\circ}, 18^{\circ}, 36^{\circ}, 54^{\circ}, 72^{\circ}, 90^{\circ}, 108^{\circ}, 126^{\circ}, 144^{\circ}, 162^{\circ}$, and $180^{\circ}$. For each viewing angle of each particular person, there are 6 video sequences. Thus, we use a total of $11 \times 124 \times 6$ or 8184 gait sequences. The dataset is separated into 2 groups. The first group contains 24 subjects and is used to construct VTM. The second group contains the rest 100 subjects and is used to evaluate performance of multiple view gait recognition.

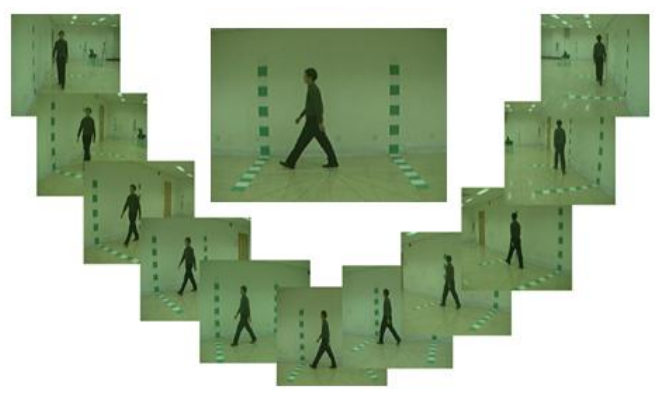

Figure 4. Sample images from CASIA gait database B.

The experiments are carried out to mainly investigate and compare the multiple view gait recognition performances for two difference approaches. Probe gait feature from one viewing angle is transformed into a feature under another view that match one of the views in gallery gait signature database. Then gait similarity is determined. The first approach uses FG feature with SVD technique referred to [7]. The second approach uses our optimized GEI feature with TSVD technique. Overview structure of the proposed techniques is illustrated in Figure 5. In addition, more experiments are conducted to investigate the improvement by using multiple-to-one view transformation (Equation (8)) through our recognition method (GEI+TSVD). The experimental results are shown in Figure 6 and Figure 7. Figure 6 illustrates first rank or the top one gait identity matching for multi-view gait recognitions. In Figure 7, Cumulative Match Scores (CMS) are used to demonstrate the multipleto-one view transformation results. The CMS value $\alpha$ corresponding to rank $r$ indicates a fraction $100 \cdot \alpha \%$ of probes whose top $r$ matches must include the real identity matches.

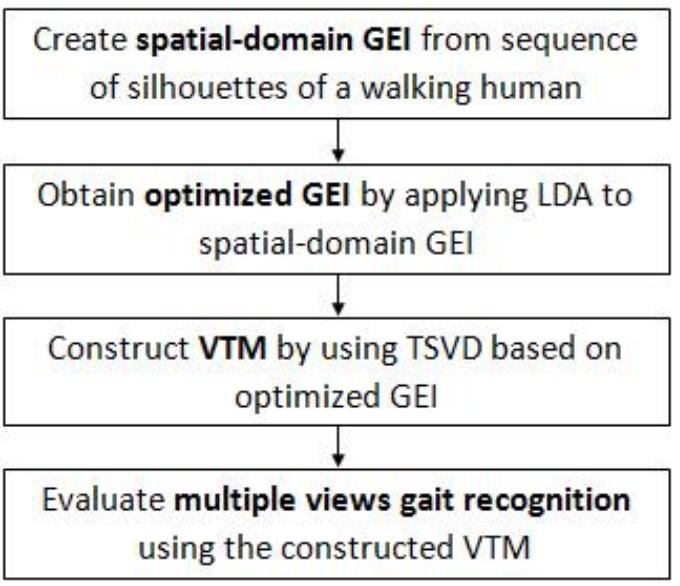

Figure 5. Overview structure of the proposed approach. 


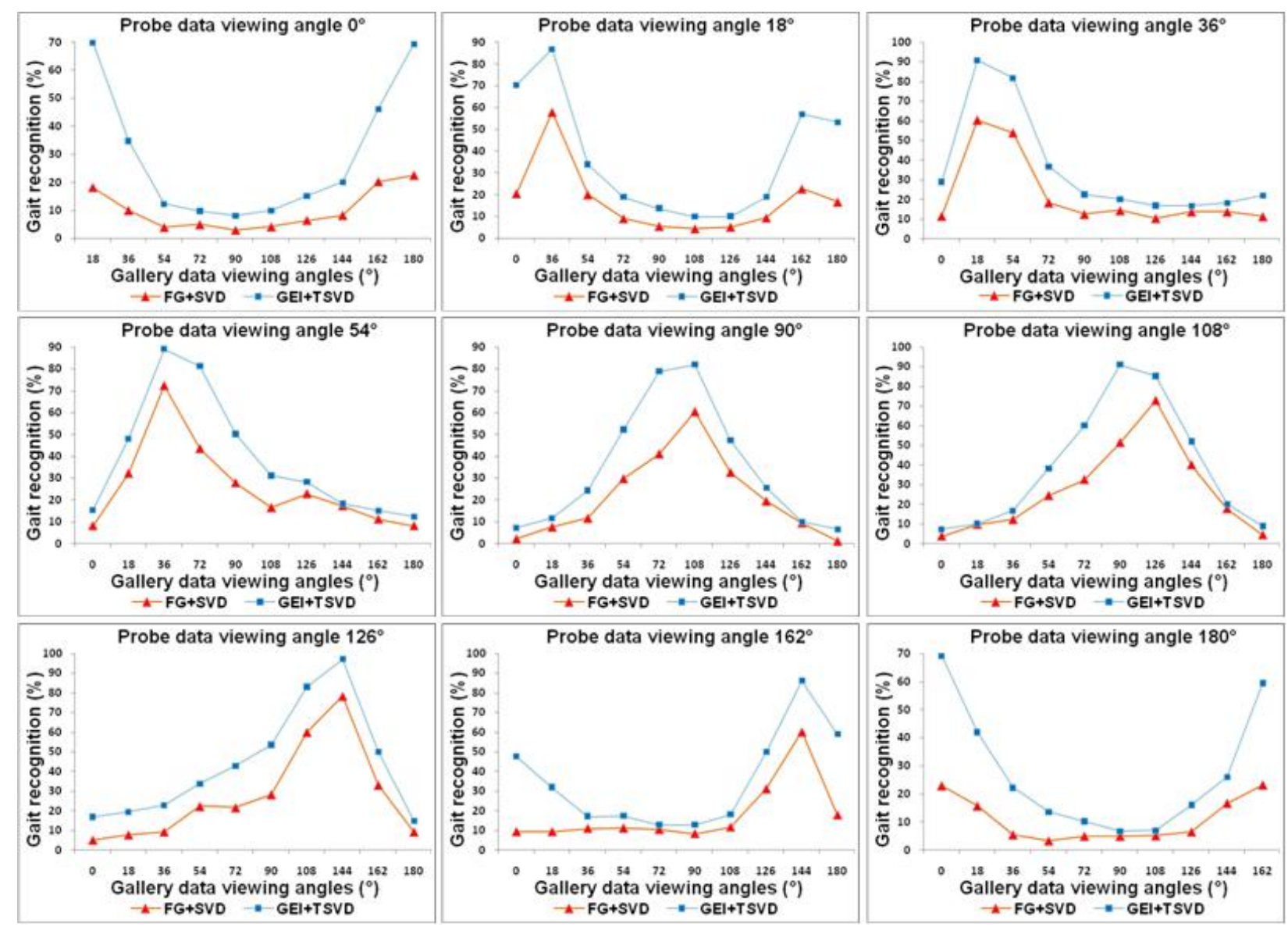

Figure 6. Comparisons of first rank multi-view gait recognition performances between the two different approaches.
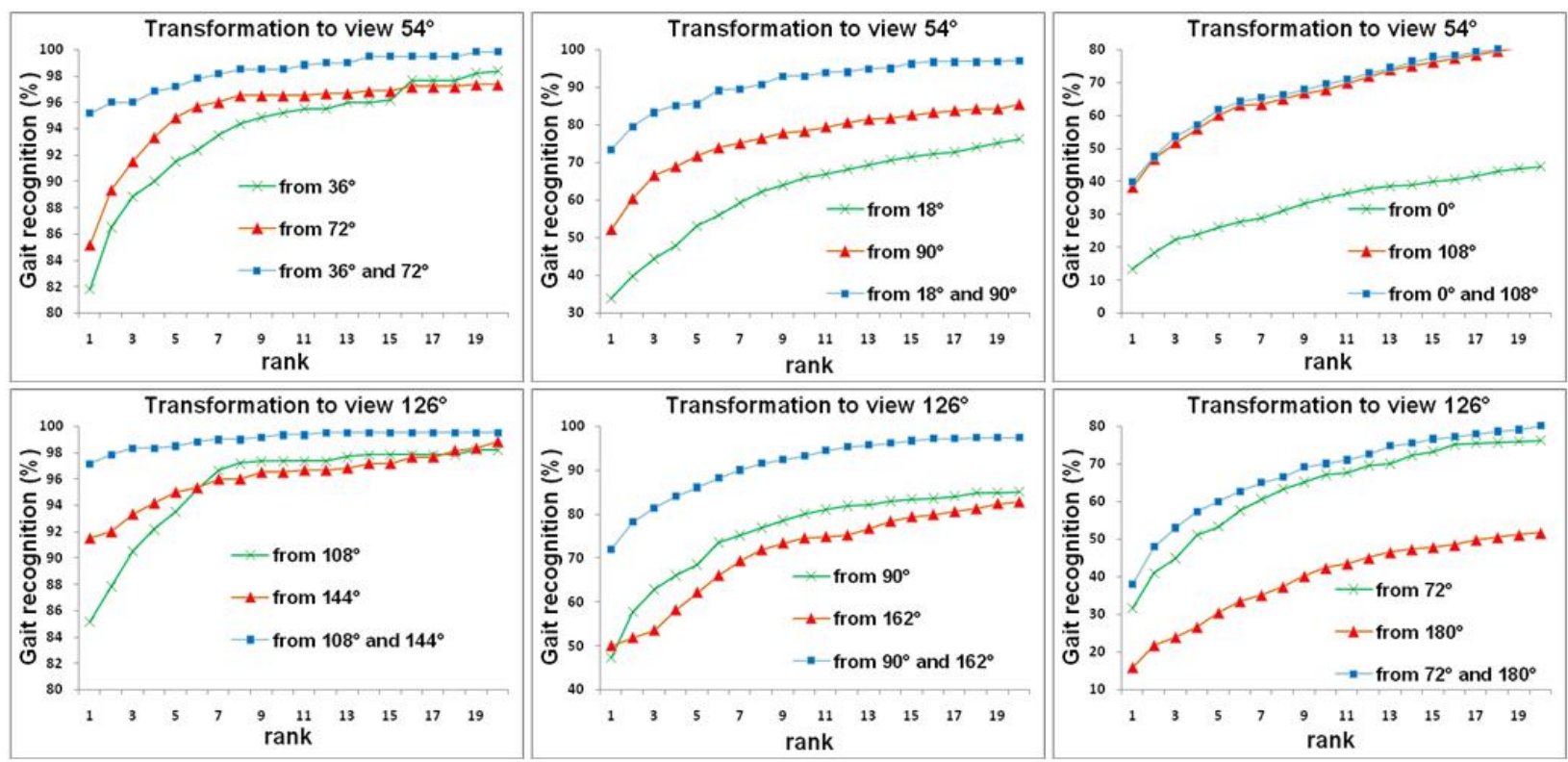

Figure 7. CMS curves for multi-view gait recognitions using multi-to-one view transformation.

\subsection{Discussion}

According to experimental results drawn in Figure 6, the proposed approach (GEI+TSVD) significantly improve multiple view gait recognition accuracy, compared with the approach (FG+SVD) referred to [7]. The proposed method achieves high performances for the close viewing angles ( $18^{\circ}$ difference), up to $90 \%$ accuracy. However, the refered method from [7] can achieve only up to approximately 
$60 \%$ accuracy. There are several key points that why our technique is able to acheive a significant improved performance.

The first key point is that compared to Frequencydomain Gait feature (FG), our spatial-domain based Gait Energy Image feature (GEI) can achieve more efficiency. As shown in Figure 2 and Figure 3, our GEI contains more non-zero or meaningful pixels than FG, which can be considered as a better feature for SVD matrix decomposition process.

The second key point is that LDA acquires optimized GEI from spatial-domain feature. This improves performances of VTM construction for multi-view gait recognition. In the experiments, LDA significantly reduces dimension on optimized GEI by $90 \%$ of spatial-domain GEI. It captures projected principal gait features with $99 \%$ significance.

The last key point is that, compare to full SVD, the TSVD can improve the multi-view gait recognition accuracy by avoiding over-fitting VTM. TSVD is strongly preferable for online real time application. The reason is that TSVD can significantly reduce size of view transformation model $40 \%$ from original size. Thus, it can achieve the reduction of computational time and memory storage.

Furthermore, it is obviously seen in Figure 6 that transform of one viewing angle of gait feature to the closer view is significantly better than to the further views. The Cumulative Match Scores (CMS) drawn in Figure 7 shows that if features for more than one viewing angles are obtained, we can transform a feature to another view more precisely.

\section{Conclusion}

This paper has proposed a new multiple views gait recognition using View Transformation Model (VTM) of optimized Gait Energy Image (GEI). We first construct a spatial-domain GEI from complete walking cycle(s). The LDA is then used to optimize the GEI. Finally, a VTM is created by applying Truncated Singular Value Decomposition (TSVD) on optimized GEI. During recognition phase, the VTM transforms gait features into the same viewing angle before the similarity measurement is conducted.

The proposed method has been verified with a large multiple view gait database. Compare to the method in [7], it can significantly improve the multiple view gait recognition performance. The proposed method does not require any camera calibration information. It can achieve the reduction on dimensions of the gait feature and size of the view transformation model. These advantages enable the proposed method to be run in real time for many surveillance applications.

\section{Acknowledgement}

1. NICTA is funded by the Australian Government as represented by the Department of Broadband, Communications and the Digital Economy and the Australian Research Council through the ICT Centre of Excellence program.

2. Portions of the research in this paper use the CASIA Gait Database collected by Institute of Automation, Chinese Academy of Sciences.

\section{References}

[1] R. Bodor, A. Drenner, D. Fehr, O. Masoud, and N. Papanikolopoulos. View-independent human motion classification using image-based reconstruction. Journal of Image and Vision Computing, 27(8):1194-1206, 2009.

[2] J. Han and B. Bhanu. Individual recognition using gait energy image, 2006. IEEE Transactions on Pattern Analysis and Machine Intelligence.

[3] F. Jean, R. Bergevin, and A. B. Albu. Computing and evaluating view-normalized body part trajectories. Journal of Image and Vision Computing, 27:1272-1284, 2009.

[4] A. Kale, K. R. Chowdhury, and R. Chellappa. Towards a view invariant gait recognition algorithm. pages 143-150, 2003. IEEE Conference on Advanced Video and Signal Based Surveillance.

[5] W. Kusakunniran, Q. Wu, H. Li, and J. Zhang. Automatic gait recognition using weighted binary pattern on video, 2009. IEEE International Conference on Advanced Video and Signal Based Surveillance.

[6] L. Lee. Gait analysis for classification. 2002. Ph.D. thesis in Massachusetts Institute of Technology.

[7] Y. Makihara, R. Sagawa, Y. Mukaigawa, T. Echigo, and Y. Yagi. Gait recognition using a view transformation model in the frequency domain, 2006. European Conference on Computer Vision.

[8] R. Sagawa, Y. Makihara, T. Echigo, and Y. Yagi. Matching gait image sequences in the frequency domain for tracking people at a distance, 2006. Asian Conference on Computer Vision.

[9] A. H. Sahoolizadeh, B. Z. Heidari, and C. H. Dehghani. A new face recognition method using pca, lda and neural network, 2008. In Proceedings of World Academy of Science, Engineering and Technology.

[10] G. Shakhnarovich, L. Lee, and T. Darrell. Integrated face and gait recognition from multiple views. 1:439-446, 2001. IEEE Conference on Computer Vision and Pattern Recognition.

[11] A. Sharifinejad. Utilizing autoregressive truncated singular value decomposition algorithm for obtaining more efficiently compressed images, 2003. Image and Vision Computing New Zealand.

[12] A. Utsumi and N. Tetsutani. Adaptation of appearance model for human tracking using geometrical pixel value distributions, 2004. In Proceedings of the 6th Asian Conference on Computer Vision.

[13] L. Wang, T. Tan, H. Ning, and W. Hu. Silhouette analysisbased gait recognition for human identification, 2003. IEEE Transactions on Pattern Analysis and Machine Intelligence.

[14] J. Ye. Least squares linear discriminant analysis, 2007. In Proceedings of the 24th International Conference on Machine Learning. 